Meta

Journal des traducteurs

Translators' Journal

\title{
Les difficultés de la structuration sémantique du lexique
}

\section{Hans-Martin Gauger}

Volume 18, numéro 1-2, mars 1973

Actes du deuxième colloque international de linguistique et de traduction. Montréal, 4-7 octobre 1972

URI : https://id.erudit.org/iderudit/002824ar

DOI : https://doi.org/10.7202/002824ar

Aller au sommaire du numéro

Éditeur(s)

Les Presses de l'Université de Montréal

ISSN

0026-0452 (imprimé)

1492-1421 (numérique)

Découvrir la revue

Citer cet article

Gauger, H.-M. (1973). Les difficultés de la structuration sémantique du lexique. Meta, 18(1-2), 145-160. https://doi.org/10.7202/002824ar d'utilisation que vous pouvez consulter en ligne. 


\section{Les difficultés \\ de la structuration sémantique du lexique}

Le terme «structuration », le mot clef — sans aucun doute — de mon sujet, prête à équivoque. J'entends par ce terme l'effort de la linguistique de reconnaître, de « reconstruire» dans sa description, une structure qui existerait, indépendamment d'elle-même, dans la réalité de la langue. Quand on parle des difficultés qui s'opposent à une telle structuration dans le domaine du sémantisme lexical, il faut partir évidemment des efforts qui ont été entrepris dans ce sens-là depuis presque cinquante ans. Puisqu'il est impossible de passer en revue tous ces efforts - assez divergents, comme on sait - je ne parlerai que de Katz et de Coseriu, c'est-à-dire de deux théories sémantiques qui, tout en se voulant «structurales » toutes les deux, sont très différentes à beaucoup d'égards. Je les traiterai comme des cas de sémantique structurale.

Katz vient de proposer sa théorie, encore une fois, dans un livre intéressant et éloquent comme tous ses écrits ${ }^{1}$. Ce livre présente une version corrigée et très amplifiée de la théorie sémantique qu'il avait avancée avec Fodor dans un article de 1963, qui est devenu, dans la constellation particulière de la linguistique à cette époque, très rapidement fameux : c'était un peu, dans la linguistique, surtout aux États-Unis, comme l'abolition de la prohibition ${ }^{2}$. Je passe tout de suite à la critique, qui sera nécessairement un peu sommaire.

Primo : La sémantique reste, chez Katz, qui adhère à ce qu'on est convenu d'appeler la «version standard» de la théorie générativiste interprétative : les mots ne sont qu'un appendice de la syntaxe; le primat, en ce qui concerne la genèse de la phrase, est concédé, d'une manière radicale, à la syntaxe. C'est là une hypothèse, qui n'est pas vraisemblable du point de vue psycholinguistique : il faut s'attendre que les relations entre la syntaxe et les mots soient autrement complexes, et s'il y a un primat, il est plutôt du côté du lexique (je reviendrai sur ce point).

Secundo : L'idée fondamentale de cette théorie, bien qu'elle ne soit guère explicitée, est l'application rigoureuse et exclusive du principe de la compositionalité, du principe du jeu de cubes. Le phénomène linguistique est conçu comme exclusivement compositionnel : est compositionnelle la phrase, son contenu; la

1. J. J. Katz, Semantic Theory, New York, Harper \& Row, 1972.

2. J. J. Katz et J. A. Fodor, "The Structure of a Semantic Theory ", Language, vol. 39, 1963 , p. 170-210 ; cf. H.-M. Gauger, " Die Semantik in der Sprachtheorie der transformationellen Grammatik », Linguistische Berichte, 1, 1969, p. 1-18. 
«lecture dérivée » qu'il constitue est le résultat de la composition hiérarchique de ces éléments. Sont compositionnels tous les groupements de morphèmes, $y$ compris, naturellement, les mots composés et les mots dérivés ${ }^{3}$. Sont compositionnels enfin les contenus des morphèmes eux-mêmes : ils sont un complexe structuré de marques syntaxiques, de marques sémantiques et de la restriction sélective qui en résulte. C'est-à-dire : les contenus des morphèmes eux-mêmes sont conçus comme quelque chose de syntaxique. La seule différence entre la sémantique lexicale et la syntaxe proprement dite réside dans le fait que, dans le cas de la sémantique lexicale, ce ne sont pas les locuteurs qui composent les éléments; les locuteurs les trouvent, dans leur compétence, comme des unités déjà composées. On peut donc parler d'une syntaxe impérialiste, puisque la sémantique lexicale se voit réduite, elle aussi, à une sorte de syntaxe. Le rôle, qui est attribué dans ce modèle au principe de la compositionalité, me paraît excessif. On reconnaît ici le trait fondamental de cette linguistique : elle cherche à isoler le fait linguistique de sa situation psychosociale; elle conçoit la langue comme un processus essentiellement combinatoire fonctionnant en lui-même. À cet égard-là (et à d'autres d'ailleurs) on peut parler du positivisme inhérent de cette linguistique : elle envisage la langue comme une entité autonome et ne se distingue donc pas, sous cet angle-là, du structuralisme «classique » (elle en est plutôt une variante à la fois radicale et partiale).

Tertio : Les «marques sémantiques » de Katz sont des concepts : « concepts that appear as parts of senses ${ }^{4} »$. Un tel concept n'est, comme il dit, «ni une image, ni une idée mentale, ni une pensée particulière »: il est une «entité abstraite ${ }^{5}$ ». Katz renvoie ici, d'une manière un peu abrupte, au logicien Frege, qu'il cite d'ailleurs comme un précurseur de son analyse en marques sémantiques (il aurait pu en citer d'autres). Le «statut ontologique » des concepts représentés par les marques sémantiques n'est donc pas défini. Katz ne veut pas dire, quant à ces concepts, ce qu'ils sont; il ne veut pas dire ce que c'est qu'un sens, « of what kinds of things senses and meanings are ${ }^{6} \gg$. Il se contente d'affirmer qu'il serait absurde d'exiger, dès le début, de tels éclaircissements : ils ne peuvent être, à son avis, que le résultat éventuel d'une théorie déjà complète. La difficulté de cette prise de position est évidemment que nous savons déjà quelque chose sur la nature du sens lexical et que la décision préalable de Katz (et d'autres) ne permet pas de rejoindre et d'approfondir ce savoir; il faudra revenir sur ce point. L'essentiel, chez Katz, me paraît être le refus de toute interprétation psychologique. La marque sémantique en tant que «concept» est une «construction théorique » dans le sens de la doctrine du soi-disant rationalisme critique $^{7}$ ».

Quarto : La théorie de Katz n'est pas à proprement parler structurale, puisqu'elle n'oppose pas, par le procédé de la commutation, les sens qu'elle décrit à d'autres sens. Katz semble considérer l'analyse en marques sémantiques comme

3. J'ai montré l'impossibilité de cette interprétation des mots composés et dérivés dans "Durchsichtige Wörter ", Zur Theorie der Wortbildung, Heidelberg, 1971, p. 23-44.

4. Semantic Theory, p. 37 .

5. Semantic Theory, p. 38 .

6. Semantic Theory, p. 39

7. Sur l'influence et les difficultés de cette doctrine dans le domaine de la linguistique, cf. H-M. Gauger, Die Linguistik und ihr Objekt, Festschrift für W. Schulz, Pfullingen,
Neske, 1973. 
une chose donnée d'avance. Les paradigmes fonctionnels n'apparaissent nulle part dans cette théorie sémantique. Pratiquement Katz ne fait que "formaliser » des articles de dictionnaires. Cette critique a été formulée, tout de suite et à plusieurs reprises, par Coseriu ${ }^{8}$. Malheureusement, dans son nouveau livre, dans lequel il discute beaucoup avec les collègues de son pays, Katz ne peut tenir compte de cette critique, puisque, comme la plupart des linguistes des États-Unis, il ne s'intéresse guère à ce qui a été publié en Europe après Descartes et Humboldt.

Ce qui nous intéresse avant tout, dans le cadre de notre sujet, c'est l'hypothèse selon laquelle un sens lexical ne serait autre chose qu'une conglomération structurée d'unités sémantiques plus petites. Cette hypothèse n'est évidemment pas originale. On la trouve dans l'« analyse componentielle» (citée par Katz) de Godenough, Lounsbury et d'autres ${ }^{9}$. Mais on trouve la même idée, d'une manière tout à fait explicite et pleinement structurale, déjà dans les "Prolégomènes» de Hjelmslev de $1943^{10}$. Le pas décisif est que l'analyse linguistique franchit - pour ainsi dire vers le bas - le seuil du signe minimal ; elle se meut dans un domaine qui reste - en ce qui concerne le signifié - en deçà du signe. Les traits sémantiques, les sèmes, que l'analyse établit, sont conçus, chez $\mathrm{Hjelm-}$ slev, comme oppositifs : un contenu est ce qu'il est, parce qu'il n'est pas ce que sont les autres. Le modèle de cette analyse est évidemment l'analyse phonologique.

La plus subtile et la plus complète des théories qui ont été développées à partir de cette idée, me paraît être celle de Coseriu, qui est d'ailleurs, à bien des égards, très proche de celle de Pottier et de Greimas, bien que l'univers de ce dernier soit beaucoup plus opaque ${ }^{11}$. Cette théorie est plus réaliste que beaucoup d'autres : «s'il peut être certain, dit Coseriu, que le lexique est le domaine le moins structuré de la langue, cela n'empêche pas qu'il soit susceptible d'un traitement structural, du moins dans la mesure où il est structuré ${ }^{12} \%$. On ne saurait, en effet, s'exprimer d'une manière plus circonspecte. Ce réalisme se manifeste avant tout dans les sept distinctions préliminaires que Coseriu juge indispensables : mots et réalité extralinguistique ; langue primaire et métalangue ; synchronie et diachronie ; technique du discours et discours répété ; langue historique et langue fonctionnelle ; système d'une part, norme et discours d'autre part ; signification et désignation. Le résultat de toutes ces distinctions est précisément une réduction considérable, dans le domaine du lexique, de ce qui est «susceptible d'un traitement structural». Geckeler, élève de Coseriu, a publié une thèse excellente, qui expose, en se basant sur cette théorie, le champ lexical des adjectifs désignant l'âge en français moderne ${ }^{13}$. Il s'agit là, à mon avis, dans le domaine

8. Par exemple : «Una lengua no es sólo un conjunto de reglas de constitución sintagmática, inmediata o mediata, sino también, y ante todo, un sistema de paradigmas functionales (Coordinación latina y coordinación románica, Actas del III Congreso español de estudios clásicos, III, Madrid, 1968, p. 38). Cf. E. Coseriu, Sprache, Strukturen und Funktionen, Tübingen, TBL, 1970, p. 213-224.

9. Cf. G. Wotjak, Untersuchungen zur Struktur der Bedeutung, Munich, Hueber, 1971, p. $110-112$.

10. Cf. H. Geckeler, Zur Wortfelddiskussion, Untersuchungen zur Gliederung des Wortfeldes «Alt-Jung-Neu» im heutigen Französisch, Munich, Fink, 1971, p. 418-432.

11. Cf. surtout E. Coseriu, Einführung in die strukturelle Betrachtung des Wortschatzes, Tübingen, TBL, 1970, et H. Geckeler, op. cit., p. 177-204.

12. Pour une sémantique diachronique structurale, TLL 2(1), 1964, p. 148.

13. Cf. note 10 . 
du lexique, de l'étude structurale la plus satisfaisante, la plus complète aussi du point de vue empirique, qu'on ait pu lire jusqu'à maintenant.

Il est évident, que cette théorie lexicale échappe - à bien des égards à la critique que nous avons formulée contre Katz. Je me concentre sur son hypothèse fondamentale : un sens déterminé, le sémène d'un lexème, est une structure d'unités plus petites : il n'est autre chose qu'une structure de sèmes ${ }^{14}$. Il est évident, que je ne pourrai qu'esquisser quelques arguments quant à cette hypothèse, dans laquelle, d'ailleurs, beaucoup de théoriciens ne verraient déjà plus une hypothèse, mais simplement une description des faits sémantiques correspondants. Je pense cependant que cette hypothèse est loin d'être vérifiée et reste une hypothèse, c'est-à-dire une «supposition que l'on fait d'une chose possible ou non et dont on tire une conséquence $\gg$ (Petit Larousse).

Le modèle de l'analyse en sèmes est l'analyse phonologique. - Mais où se trouve ici le parallélisme? Les sèmes correspondent-ils aux phonèmes ou aux traits phonétiques distinctifs, aux phèmes? Entre les phonèmes et les sèmes il y a une différence considérable : les phonèmes sont linéaires et peuvent, par conséquent, être identifiés beaucoup plus facilement, bien que nombre d'incertitudes subsistent. Les sèmes par contre ne sont pas linéaires : ils apparaissent simultanément en un point de la chaîne sonore, marqué par un ou plusieurs phonèmes, c'est-à-dire par le signifiant du lexème. Il y a donc là une asymétrie remarquable : en franchissant le seuil du signe minimal, on arrive, dans le domaine du signifiant, à deux unités : une unité linéaire, le phonème, et une unité non linéaire, le phème ; dans le domaine du signifié cependant, on arrive à une seule unité, le sème, qui n'est déjà plus linéaire. Mais il y a aussi des différences considérables entre les phèmes et les sèmes, bien qu'ils soient tous les deux non linéaires : d'abord les sèmes sont beaucoup moins faciles à saisir et à formuler linguistiquement que les phèmes, qui peuvent être décrits du point de vue articulatoire ou acoustique et où il ne s'agit très souvent que de l'alternative présence ou absence d'un trait : sourd' n'est en effet autre chose que non sonore, tandis que le sémème « jeune » se distingue du sémème de son antonyme "vieux» non seulement par la négation d'un certain sème contenu dans «vieux»; «jeune » n'est pas simplement «non vieux », et «vieux» n'est pas simplement «non jeune». - Deuxièmement la différence quantitative entre les phèmes et les sèmes est tellement grande qu'elle doit se transformer inévitablement en une différence qualitative : les phèmes ne composent que quelques douzaines d'unités, les sèmes beaucoup de milliers. Troisièmement les phèmes sont purement intralinguistiques, les sèmes cependant, qui ne sont pas des signes (puisqu'il leur manque un signifiant), partagent avec les signes le caractère intentionnel : ils sont dirigés vers quelque chose en dehors d'eux-mêmes. C'est pourquoi il faut parler, quand on décrit des sèmes, d'une manière pratiquement inextricable, d'éléments linguistiques et extralinguistiques à la fois. Il se manifeste ici une difficulté plus générale : elle me semble être une des plus ardues que présente le traitement structural du lexique. Il faut en parler tout de suite, et ce n'est certainement pas la première fois qu'on en parle.

14. Cf. le très intéressant article de G. Hilty, "Bedeutung als Semstruktur " (Vox románi$c a, 30(2), 1971$, p. 242-263), qui contient dans son titre même cette hypothèse. Nous avons mené, $M$. Hilty et moi, une discussion amicale sur cet article dans le cahier à paraître de Vox románica. Je reprends ici quelques-uns de mes arguments. 
Il est extrêmement difficile, sinon impossible, de faire le départ, dans le lexique, de ce qui peut être considéré comme savoir linguistique et de ce qui est connaissance $d u$ monde dans lequel nous vivons. Coseriu distingue entre le lexique structuré, proprement linguistique, et le lexique non structuré, qu'il appelle «nomenclature». Cette distinction est importante, mais il est difficile de trouver des critères dans nombre de cas concrets; et d'ailleurs : les contenus lexicaux ne doivent-ils pas être considérés, dans leur ensemble, comme un vaste réseau de connaissances, plus ou moins complètes, préscientifiques (ou postscientifiques) sur le monde ? Où trouver, dans cet amas confus et parfois difficilement saisissable, ce qu'il pourrait y avoir de proprement linguistique ?

Les définitions suivantes sont choisies, sans mauvaise foi, du Dictionnaire fondamental de Gougenheim et du Petit Larousse :

arbre : "plante très haute, avec des branches $x$

impôt : «somme que l'on doit payer chaque année à l'État »

maison : «bâtiment (en pierres, en briques, en bois, etc.) où l'on habite"

libre : "qui peut faire ce qu'il veut»

bleu : " qui est de la couleur du ciel quand il fait très beau »

rossignol : "oiseau qui chante très bien "

dormir : "se reposer en fermant les yeux " rire : "faire un mouvement de la bouche et des joues (en découvrant un peu les dents) qui montre qu'on s'amuse "

venir : " aller dans une ville, chez quelqu'un» arbre: "plante ligneuse dont la tige, ou tronc, fixée au sol des racines est nue à la base et chargée de branches et de feuilles à son sommet *

impôt : "contribution exigée pour assurer le fonctionnement de l'État et des collectivités locales »

maison : «construction destinée à l'habitation humaine

libre : "qui a le pouvoir d'agir ou de ne pas agir; qui ne subit pas la domination étrangère... »

bleu : " de couleur d'azur, de la couleur du ciel sans nuages»

rossignol : "oiseau passereau à plumage brun clair séjournant l'été dans nos bois et parcs, et dont le mâle est un chanteur remarquable * dormir : « reposer dans le sommeil»

rire : " marquer un sentiment de gaieté par un mouvement des lèvres, de la bouche, et souvent avec bruit"

venir : "se transporter d'un lieu dans un autre; se rendre chez quelqu'un "

Ces définitions, qui se veulent linguistiques et non encyclopédiques, se proposent de décrire le sens de ces mots, mais ce qu'elles décrivent en vérité n'est autre chose que les réalités extralinguistiques que ces mots désignent, et la description est nécessairement très rudimentaire. La différence entre les définitions du Petit Larousse et celles de Gougenheim ne consiste que dans le fait que le premier va évidemment plus loin et dépasse ainsi, très souvent, les connaissances que l'on trouve, en général, chez le locuteur moyen ${ }^{15}$.

Quel peut être l'intérêt linguistique de telles descriptions? Qu'a-t-on fait quand on décrit le sens de sel par «petits grains blancs qu'on met dans les aliments pour leur donner du goût » ou celui de dent par «un des petits os qui se trouvent sur le devant de la bouche \& (Gougenheim) ? Quel peut être l'intérêt linguistique de « formaliser 》 de telles définitions en les décomposant en sèmes et en ordonnant ces sèmes, comme le font Katz et d'autres (non pas Coseriu), selon le schéma

15. Cf. H.-M. Gauger, Wort und Sprache, Tübingen, Niemeyer, 1970, p. 84-87. 
genus/species, d'ailleurs très problématique, de la logique très traditionnelle (ce schéma a déjà été employé, à d'autres fins, par Platon) ?

Ici cependant, il faut faire une restriction importante : il y a beaucoup de mots dont le sens est, pour ainsi dire, plus linguistique. C'est le cas, quand les différences entre les choses ne s'expliquent que par le fait qu'une langue particulière les considère comme différentes. Aller et venir, par exemple, appartiennent à ce type. Aucune science ne peut nous expliquer, plus exactement que ne le fait déjà la langue, ce que c'est que la «chose » venir. Dans ces cas-là, l'intérêt linguistique d'une description ne fait pas de doute. Mais comment séparer, par des critères rigoureux, ces mots-là des autres ? Et d'ailleurs, même en ce qui concerne ces contenus lexicaux plus «linguistiques», il faut parler de la réalité signifiée elle-même, car il y a là aussi des éléments qu'on doit considérer comme une sorte de «connaissance » de la chose telle qu'elle est vue par la langue.

Les difficultés que nous trouvons ici, sont la conséquence du fait que la langue n'est pas une chose «en elle-même et pour elle-même » pour reprendre la fameuse formule, qui ne provient pas, comme on sait, de l'éminent linguiste genevois et qui lui fait tort, puisqu'il voulait qu'on étudie le signe précisément « au sein de la vie sociale ${ }^{16}$ ». Le trait essentiel de la langue est précisément qu'elle représente quelque chose en dehors d'elle-même. La philosophie a parlé ici de son «intentionnalité », terme propagé par Edmund Husserl et créé par Franz Brentano. Or, cette intentionnalité de la langue est ancrée dans ses mots : l'univers est présent dans la langue - non pas exclusivement, mais avant tout par son lexique ${ }^{17}$. C'est dans ce sens-là que le lexique est la partie centrale et constitutive de la langue et qu'il faut lui concéder le primat sur la syntaxe. La langue n'est pas, comme veulent le faire croire les générativistes, un phénomène essentiellement combinatoire. Elle contient, en le reflétant, le monde en lui-même dans la mesure où celui-ci nous est accessible, et puisqu'elle est liée à la réalité psychosociale des sujets qui l'emploient, elle est déterminée, déjà en tant que compétence et encore plus dans ses énoncés concrets, par nombre de facteurs en dehors d'elle-même. Voilà pourquoi, en définitive, une linguistique, qui s'oriente exclusivement selon le principe compositionnel, est vouée à l'échec.

$\mathrm{Ce}$ qui est en jeu ici n'est autre chose que la notion de «compétence linguistique » elle-même. La compétence linguistique ne peut être isolée, en tout cas dans le lexique, d'une compétence beaucoup plus générale qu'on a appelée - et c'est probablement une notion encore trop étroite - «compétence communicative » (c'est le terme qu'emploie le philosophe Habermas, dont je n'accepte pas, d'ailleurs, dans leur ensemble, les idées linguistiques) ${ }^{18}$. Pour le moment, il s'agit seulement de voir que ce qu'on appelle la «compétence linguistique» n'est ni un objet autonome, ni même un objet isolable méthodiquement.

Coseriu insiste beaucoup sur l'importance de la distinction entre signification et désignation, qui joue un grand rôle, comme on sait, dans la logique tradition-

16. En vérité la position de Saussure est ici curieusement ambiguë ; cf. H.-M. Gauger, Die Linguistik und ihr Objekt, Festschrift für W. Schulz, Pfullingen, Neske, 1973.

17. Cf. H.-M. Gauger, Wort und Sprache, p. 24-25, p. 50-51.

18. J. Habermas, "Einführende Bemerkungen zu einer Theorie der kommunikativen Kompetenz ", dans J. Habermas et N. Luhmann, Theorie der Gesellschaft oder Sozialtechnologie, Francfort, Suhrkamp, 1971, p. 101-141. 
nelle et moderne. Il la considère comme «fondamentale pour la sémantique structurale et la linguistique fonctionnelle en général ${ }^{19}$ \%. La signification et la désignation sont, pour lui, «deux fonctions absolument différentes ${ }^{20}$ \%. Cette distinction vise d'abord à la différence entre le signifié et la chose. Dans ce sens-là, elle est sans aucun doute absolument indispensable et d'ailleurs très ancienne, comme l'indique Coseriu lui-même. Il est vrai que Bloomfield, induit en erreur par sa psychologie behavioriste, ne l'a pas vue, et que le générativisme est resté assez bloomfieldien à cet égard, parce qu'il parle très souvent d'une identité de signification, quand, en réalité, il ne s'agit que d'une identité de la chose signifiée ${ }^{21}$. Reprenons encore une fois l'exemple tant de fois invoqué de Husserl : le vainqueur de Iéna, le vaincu de Waterloo; nous avons ici : identité de désignation (reference) et diversité de signification (meaning) ${ }^{22}$. Dans ce sens-là, la distinction est aujourd'hui largement acceptée.

Mais Coseriu semble penser, quand il parle de désignation, encore à autre chose, à un élément de l'acte linguistique lui-même, à une entité sémantique à côté de la signification. La désignation représenterait « la connaissance des choses extérieures ». On aurait donc, pour ainsi dire, deux plans dans les contenus sémantiques : le plan de la signification, qui serait linguistique dans le sens étroit, et le plan de la désignation, qui serait à la fois linguistique et extralinguistique : linguistique, parce qu'il appartient à la langue; extralinguistique, parce qu'il représente - dans la langue - «la connaissance des choses ». La signification est constituée par les rapports entre les signifiés, tandis que la désignation est, selon Geckeler, « le rapport (ou la mise en rapport) du signe linguistique total avec les « objets » de la réalité extralinguistique ${ }^{23} \gg$. La signification serait ainsi une condition précédant la désignation : "une possibilité ou virtualité de désignation ${ }^{24} \gg$. Prise dans ce sens-là, la distinction entre signification et désignation est un élément spécifique de la théorie de Coseriu, et elle ne serait certainement pas acceptée par beaucoup de théoriciens, ce qui ne prouve pas, évidemment, qu'elle soit inacceptable.

Chez Coseriu, cette distinction est établie, si je ne me trompe, en partant déjà de l'hypothèse structurale : les significations sont structurées, les désignations ne le sont pas; le critère de la distinction est précisément la structuration ou la non-structuration. II faudrait cependant qu'on soit à même d'expliciter la différence entre ces deux «plans» sémantiques en dehors de l'hypothèse structurale. Sinon, l'argument ressemble trop à une pétition de principe : pour faire de la sémantique structurale, il faut distinguer entre signification et désignation; or, la signification - en tant que rapport entre signifiés - est ce qui est structuré ; le reste, le non structuré, est désignation.

19. E. Coseriu, "Bedeutung und Bezeichnung im Lichte der strukturellen Semantik», dans Sprachwissenschaft und Ubersetzen, Commentationes Societatis Linguisticae Europae III, Munich, Hueber, 1970 , p. 2.

20. Cf. H. Geckeler, op. cit., p. 38

21. C. Rohrer cherche à intégrer cette distinction dans la théorie générativiste (Funktionelle Sprachwissenschaft und transformationelle Grammatik, Munich, Fink, 1971, p. 88-99).

22. E. Husserl, Logische Untersuchungen, Zweiter Theil: Untersuchungen zur Phänomenologie und Theorie der Erkenntnis, Halle, Niemeyer, 1901, p. 47 ; cf. H. Geckeler, op. cit., p. 80 .

23. H. Geckeler, op. cit., p. 81.

24. Cf. H. Geckeler, op. cit., p. 83 
Mais il $\mathrm{y}$ a encore d'autres problèmes que je ne peux qu'énumérer ici. D'abord : comment est-ce qu'on arrive, dans un acte de parole, de la signification à la désignation ? Ensuite : comment arriver, dans l'analyse sémantique, de la désignation à la signification (car on ne trouve, comme matériel đe cette analyse, que des désignations) ? Finalement : comment concevoir la signification indépendamment de tout élément de désignation? La signification n'est-elle pas, déjà en tant que telle, désignation? Comment séparer la signification, comme élément purement et proprement linguistique, de la désignation en tant que « connaissance des choses »? Chez Coseriu le domaine des significations apparaît comme le hortus conclusus des structures; ce qui ne veut pas entrer dans les structurations oppositives, est rejeté aux ténèbres extérieures des désignations. Je pense donc que cette distinction intéressante présente encore des problèmes. Il est possible, évidemment, que je me trompe, mais alors il sera possible de me dire pourquoi.

À mon avis, le mot se réfère — déjà comme élément virtuel de la langue à une chose. Non pas à une chose individuelle, mais, pour parler avec la logique, à une «classe »de choses. Mais ce recours à la logique n'est pas indispensable ; il suffit de dire que des mots comme arbre, vieux, dormir désignent « des choses comme $X »$, « des cas de $X$ » c'est-à-dire des objets, réels ou imaginés, qui apparaissent, en vertu d'un certain nombre de traits communs, comme des exemplaires, des cas d'une seule et même chose. Il faut se rendre compte d'ailleurs que ce qui est considéré comme une seule et même chose n'est pas établi en dehors de la langue : une chose n'est une chose que par l'intermédiaire d'une langue historique déterminée.

Quant au contenu du mot «lié » à sa forme phonétique, il me paraît très important de ne pas le confondre avec ce que le mot signifie. Ce contenu est le moyen par lequel le mot signifie : il fait que la forme phonique soit un mot. La signification n'est pas une chose qui se meut dans le mot lui-même. Le véritable «signifié », si l'on s'en tient au sens grammatical de ce terme, n'est pas ce qu'on est convenu d'appeler ainsi depuis Saussure : le véritable «signifié » est la chose elle-même. Ce qu'on appelle le «signifié » n'est que la condition nécessaire et suffisante qui permet au mot de signifier une chose. C'est d'ailleurs ce qu'exprime clairement la phrase scolastique souvent citée : «verba [ou voces; il y a là une difficulté que je laisse de côté] significant res mediantibus conceptibus ». Ce sont donc les res qui sont ici les objets, les significata, du significare des verba. Si je remplace «concept» par «contenu», cette phrase traduit exactement ce que je veux dire. Le schéma suivant se réfère au mot virtuel :

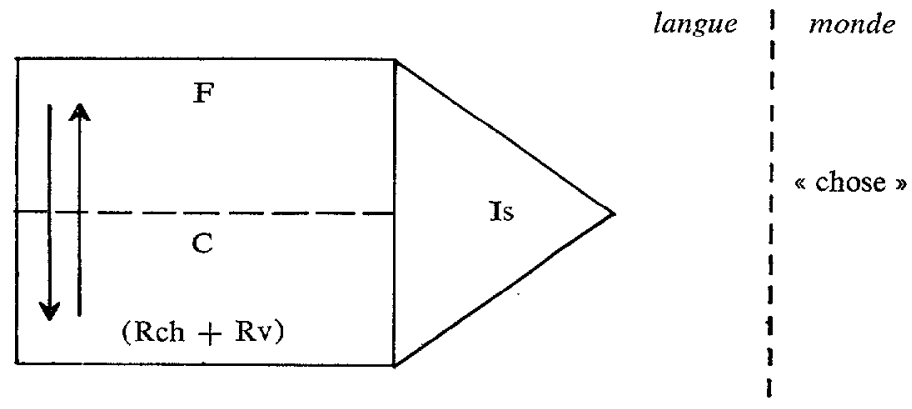


La forme phonique (F) est donc «liée» - dans et pour la conscience des locuteurs - à un contenu (C) qui rend possible l'intentionnalité signifiante (Is) du mot. Les deux flèches symbolisent «le rapport d'évocation réciproque» (Ullmann) entre $\mathrm{F}$ et $\mathrm{C}$. La séparation entre «langue » et «monde » est délicate, naturellement, puisque la langue, elle aussi, fait partie du monde, sans parler du problème philosophique attaché à la notion de monde ou d'univers conçue d'abord par les philosophes grecs. Mais le linguiste a le droit, quelquefois, d'être plus ingénu que le philosophe, surtout parce qu'il ne cherche qu'à expliciter les. conceptions intuitives qu'ont les locuteurs et qui sont une partie intégrante de leur « compétence » même.

Le contenu sémantique (C) du mot nous intéresse ici avant tout. Il consiste en deux éléments : une représentation de la chose signifiée (Rch) en tant que «classe », et une représentation du mot lui-même, une représentation verbale (Rv). Par «représentation de la chose »j'entends le savoir, en général intuitif, du locuteur qui lui permet d'employer un mot correctement : il sait quels éléments doivent être réunis pour qu'une chose déterminée puisse être désignée par tel ou tel mot. C'est un savoir qu'il a acquis dans la praxis du langage et qui est continuellement confirmé ou corrigé par elle. Il n'est d'ailleurs pas rare, qu'on se rende compte, en parlant ou en écrivant, d'un certain décalage entre la représentation attachée à un mot et la chose individuelle qui tombe sous nos sens ou que nous concevons : on ne sait pas alors exactement, si tel ou tel mot convient vraiment pour désigner cette chose ; on compare, dans ce cas, ce qu'on voit ou ce qu'on conçoit avec les représentations de choses attachées aux mots qu'on veut employer. La représentation du mot s'attache surtout à son caractère phonique; l'expression «image acoustique », employée par Saussure pour caractériser le signifiant, est ici convenable. Souvent, par exemple dans le cas des onomatopées, la représentation verbale n'est pas nettement séparable de la représentation de la chose. La représentation verbale contient aussi le savoir qu'a acquis le locuteur quant au caractère spécifique du mot lui-même : il sait — et cela aussi fait partic de sa «compétence» - qu'il s'agit par exemple d'un mot vieilli, d'un mot nouveau, d'un mot rare, argotique, solennel, d'un mot employé surtout par les femmes, par les ouvriers, d'un mot poétique, noble, trivial, etc. Voilà donc un champ très vaste que la description ne doit pas passer sous silence. On se rend compte ici, d'ailleurs, que la langue n'est pas simplement «compétence »; elle est une «compétence » en partie consciente d'elle-même.

Cette conception du mot et de son contenu, que j'ai développée plus amplement dans mes livres Wort und Sprache et Zum Problem der Synonyme, correspond à celle qu'a esquissée Freud dans quelques-uns de ses écrits ${ }^{25}$. On sait que Freud attribue au langage un rôle central dans la constitution psychique et que la cure psychanalytique, qui est à la base de sa psychologie, a un caractère érninemment linguistique : «la seule chose qui se passe dans le traitement analytique est un échange de paroles entre l'analysé et le médecin ${ }^{26}$. $\gg$ Il est

25. H.-M. Gauger, Wort und Sprache, Sprachwissenschaftliche Grundfragen, Tübingen, Niemeyer, 1970 ; H.-M. Gauger, Zum Problem der Synonyme, avec un résumé en français : Apport au problème des synonymes, Tübingen, TBL, 1972.

26. S. Freud, Gesammelte Werke, XI, p. 9. 
curieux de constater que ni la linguistique ni même la psycholinguistique ne se sont guère intéressées, jusqu'à maintenant, à la psychanalyse, bien qu'il y ait là, sans aucun doute, beaucoup d'éléments intéressants pour la compréhension du langage (il est clair, d'autre part, que l'œuvre de Freud ne contient pas une théorie toute faite du mot ou du langage : il s'agit d'éléments qui pourraient servir de fondement à une telle théorie, c'est-à-dire il faudrait en faire quelque chose linguistiquement) ${ }^{27}$. Je renvoie à un passage de l'article «L'inconscient » (1915), article très important, parce qu'il marque un tournant intéressant dans les conceptions de son auteur ${ }^{28}$. Freud explique ici, que ce qui donne la qualité de «conscient » à un contenu psychique est précisément le fait qu'il est attaché à une représentation verbale : il dit qu'un contenu, une représentation de chose, est «occupé » par une représentation verbale. La conscience serait donc, selon Freud, intimement liée à la langue et plus particulièrement au lexique : le langage est la condition sine qua non de la conscience. Les contenus inconscients du psychisme (on connaît l'importance que Freud leur attribue) sont ceux précisément qui ne sont pas «occupés» par des représentations verbales, ce qui ne veut pas dire, d'ailleurs, que le «système» de l'inconscient soit sans langage : il est caractérisé par un autre langage, «archaïque et plus primitif », qui réapparaît dans les symptômes névrotiques et dans la «régression formelle » du langage onirique.

Le mot consiste donc, pour Freud, en une « double représentation »: une représentation de la chose et une représentation verbale principalement auditive. Quant à la représentation de la chose, qui nous intéresse ici particulièrement, Freud la définit comme une «image mémorielle de choses » (Sacherinnerungsbilder), ou, d'une manière plus prudente, comme un ensemble de «traces mémorielles plus éloignées et dérivées d'images de choses» (entferntere und von ihnen abgeleitete Erinnerungsspuren). La notion d'image est ici essentielle; elle n'est d'ailleurs certainement pas nouvelle (nous la trouvons déjà chez Aristote, quand il définit, dans un passage fameux, les $\pi a \theta \eta \mu \alpha \tau a \tau \eta \varsigma$ s $\psi \nu \chi \eta \varsigma$ comme des $\delta \mu o \iota \omega \mu \alpha \tau a$ : les idées ou les représentations, dont les sons sont les signes, sont les images des choses) ${ }^{29}$. Ce que nous appelons le «contenu $» d u$ mot aurait donc, selon cette conception, un caractère (plus ou moins) visuel. Je ne peux parler ici d'autres éléments de la théorie psychologique de Freud qui renforcent cette prise de position, comme, par exemple, le langage visuel du rêve (le rêve «traduit», selon Freud, un énoncé du langage normal en un langage visuel et fait ainsi de cet énoncé une espèce de « rébus 》).

C'est ici, de toute façon, que se manifeste clairement la différence entre cette conception et celle du structuralisme et du générativisme. Cette dernière voit le contenu comme quelque chose d'essentiellement (ou même d'exclusivement)

27. Il faut citer ici avant tout P. Ricœur, De l'interprétation, Paris, Seuil, 1965, et J. Lacan, Ecrits I, et II, Paris, Seuil, 1966, et 1971 : cf. aussi G. Jappe, Ueber Wort und Sprache in der Psychoanalyse, Francfort, Fischer, 1971. Je suis en train de préparer un livre sur Sprache und Sprechen im Werk Sigmund Freuds.

28. S. Freud, Gesammelte Werke, X, p. 300. Freud se concentre, dans sa phase tardive, beaucoup plus sur le rôle du langage, dans la constitution du Ego; au début il s'était intéressé avant tout aux relations entre le langage et le Id.

29. De interpretatione $(16 \mathrm{a})$. 
conceptuel, tandis que Freud y voit quelque chose d'essentiellement visuel ou quelque chose qui reste lié, plus ou moins étroitement, au visuel. Je crois qu'il faut, en effet, considérer le contenu d'un mot comme quelque chose qui est à mi-chemin entre l'image et le concept : il y a en lui, dans un degré variable, à la fois des éléments visuels et des éléments conceptuels. Voilà pourquoi le terme de représentation, qui est une espèce de compromis entre les deux, me paraît ici le mot juste. Il est intéressant par ailleurs de constater que la psycholinguistique néo-behavioriste, dont le point de départ est absolument différent, a développé une idée analogue, largement acceptée dans son propre camp, celle du « $\mathrm{r}_{\mathrm{m}} »$ (mediating response ou representational mediation process), qui est défini comme representational, most easily detachable parts of responses ou, plus exactement, comme leurs possibles cerebral representations. Il s'agit là d'une construction hypothétique (hypothetical construct with possible existential properties) développée surtout par Osgood et Staats ${ }^{30}$.

La notion de représentation, je l'avoue, n'est pas très précise. Je pourrais me contenter de dire que la notion de concept, qui est l'élément de base des modèles structuraux, n'est pas très précise non plus (Katz dit même qu'il serait absurde d'exiger une précision préalable de la notion de «marque sémantique »). Mais la précision de nos modèles descriptifs ne doit pas être poussée au-delà de la précision de nos objets mêmes, sinon la description deviendra nécessairement inadéquate. Notre description doit rendre compte du fait que le contenu lexical, en tant qu'élément social de notre conscience, est, dans une certaine mesure, objectivement imprécis. Peut-être puis-je rappeler ici un passage d'un penseur très différent de Freud et qui n'était certainement pas - tout comme Freud d'ailleurs - un mystique : en s'efforçant de décrire le sens du mot jeu (Spiel), Wittgenstein fait l'observation suivante : «On peut dire que le concept de « jeu " est un concept aux marges floues. Mais un concept flou est-il vraiment un concept? Une photographie imprécise est-elle le portrait d'un homme? Est-il toujours avantageux de remplacer un portrait flou par un portrait précis? N'avonsnous pas besoin, justement, d'un portrait flou ${ }^{31}$ ? $\gg$ En effet, la reconstruction descriptive du contenu ne peut être plus précise que ce contenu lui-même : elle doit se contenter d'être aussi précise que possible, car la précision n'est pas un but absolu. Il serait absurde d'accuser d'imprécision celui qui constate, que l'objet de sa description est imprécis, comme il serait absurde d'accuser d'irrationalisme celui qui reconnaît le rôle de l'irrationnel dans l'histoire. Être rationnel signifie d'abord prendre les choses telles qu'elles sont.

Je ne préconise donc certainement pas des descriptions floues, mais je pense, que nos descriptions doivent rendre compte, si elles veulent être adéquates, du flou objectif, d'ailleurs variable, des contenus sémantiques, si rudimentaires souvent, en ce qui concerne leurs éléments conceptuels. Ces contenus peuvent être rudimentaires, parce qu'ils ne sont, comme je l'ai dit, que le moyen qui permet au mot de signifier, une sorte de tremplin, pour l'esprit, vers la chose. Nos descriptions doivent rendre compte aussi de la grande plasticité des contenus lexicaux,

30. Cf. Semantics, An Interdisciplinary Reader in Philosophy, Linguistics and Psychology, édit. par D. D. Steinberg et L. A. Jakobovits, Cambridge, UP, 1971, p. 487-490, 497-529. 31. L. Wittgenstein, Philosophische Untersuchungen, Francfort, 1967, p. 50, $\$ 71$. 
de leur constante ouverture à des déterminations nouvelles, qui est la qualité de leur défaut conceptuel. Les marques sémantiques chez Katz, par exemple, sont comme une réunion siégeant à huis-clos, tandis qu'en réalité, comme l'a remarqué Freud également, «la représentation de la chose est un complexe représentationnel ouvert ${ }^{32} \gg$.

La difficulté la plus grande qui s'oppose à la structuration sémantique du lexique est donc certainement celle qui est donnée par la nature même du contenu lexical. Ce contenu ne peut être qu'une entité psychique, et si la linguistique veut être, comme dit Chomsky, «a chapter of human psychology ${ }^{33}$, elle ne peut être libre dans ses constructions théoriques. La sémantique lexicale ne peut faire semblant de ne rien savoir de la nature du contenu lexical; en réalité, nous en savons quelque chose, et il s'agit de compléter et d'approfondir ce savoir. Selon l'hypothèse structurale, il faudrait supposer qu'il $\mathrm{y}$ ait, au-dessous de la représentation psychique, une autre réalité, plus profonde, plus « réelle» peut-être aussi : la structure oppositive. Cette hypothèse est loin d'être vérifiée; elle ne me paraît pas vraisemblable en partant des données que nous venons d'esquisser.

Quelle sera la tâche de la sémantique lexicale ? Elle doit décrire les éléments réunis dans un contenu lexical (pour cela, elle doit se servir des moyens techniques extraordinaires aujourd'hui disponibles). Elle doit reconstruire les conditions qui doivent être remplies, pour qu'un mot déterminé puisse être employé dans un contexte et une situation déterminés. Ces conditions sont à la fois extralinguistiques et intralinguistiques, car la sémantique ne peut partir, comme Katz le propose, d'un locuteur « idéal » qui serait en pleine possession de sa compétence linguistique, mais qui serait, en ce qui concerne les faits non linguistiques, la victime d'une amnésie ${ }^{34}$. Il est certain - car il n'y a pas d'autres possibilités - que la description doit procéder par la voie de l'analyse. Sans aucun doute, on peut discerner dans un contenu lexical des traits distinctifs, des sèmes; évidemment, ce qu'on appelle un sémème n'est pas une entité compacte, «monolithique ou atomiquee ${ }^{35}$ ». On nous présente ici une fausse alternative, sans envisager qu'il y a, entre «monolithique » d'une part et «structure de sèmes» de l'autre, une troisième possibilité. Le contenu contient des sèmes, et il doit, par conséquent, être analysé en sèmes. Mais le contenu n'est pas une conglomération ou une structure de sèmes. C'est-à-dire : l'analyse en traits distinctifs n'atteint pas, pour complète qu'elle soit, la totalité de la représentation, parce que cette totalité est en quelque sorte excédente quant à la somme ou à la hiérarchie des sèmes qu'on peut discerner en elle : dans le cas de cette représentation, comme dans l'autre cas d'ailleurs, le tout est plus (et est autre chose) que la somme de ses parties. Il faut revenir ici au caractère plus ou moins visualisé de la représentation de la chose ; ce caractère lui donne une espèce de totalité unitaire qui échappe précisément à toute reconstruction par des traits différentiels. Voilà pourquoi une analyse en

32. S. Freud, Zur Auffassung der Aphasien, Leipzig-Vienne, Deuticke, 1891, p. 79. Ce livre préanalytique de Freud est particulièrement intéressant quant à sa conception du langage.

33. N. Chomsky, Language and Mind, New York, Harcourt, 1968, p. 59 ; cf. p. 1 et p. 24.

34. Katz-Fodor : "The theory... interprets discourses as would a fluent speaker afflicted with amnesia for non-linguistic facts but not with aphasia » (Structure, p. 490).

35. Katz, Structure, p. 37. 
sèmes ne peut atteindre pleinement son but. Elle devra donc reconnaître son caractère inévitablement approximatif. L'analyse en sèmes telle qu'elle est pratiquée par le structuralisme n'est donc pas fausse ; elle contient un élément important de vérité, elle n'est pas, cependant, la vérité entière.

De la même façon le principe de l'opposition n'est certainement pas faux. Ce principe joue un grand rôle dans la différenciation sémantique des contenus lexicaux ; bien qu'il ne joue pas le rôle constitutif et exclusif — «dans la langue il n'y a que des différences $\gg$ - que le structuralisme classique lui avait conféré. L'observation selon laquelle « on ne se définit qu'en s'opposant 》 vaut aussi pour la sémantique lexicale. Le principe du vocabulaire étant justement la distinction, il est évident que les contenus lexicaux se précisent mutuellement : ils se rendent mutuellement plus denses et plus riches, un processus que l'on peut observer, d'une manière paradigmatique, pendant l'apprentissage de la langue. Il ne s'agit donc pas de retomber dans ce qu'on avait coutume d'appeler l'atomisme. Là aussi l'approche structuraliste contient un élément important de vérité.

La description se rendra compte aussi que beaucoup de traits distinctifs ne peuvent être formulés linguistiquement d'une manière claire et distincte, ce qui serait nécessaire pour qu'il y ait une véritable structure. On ne pourra pas non plus, dans tous les cas, séparer nettement les mêmes « dénotatifs » des sèmes «connotatifs », c'est-à-dire les sèmes $d$ '《 appel » et $d$ '« expression», selon le schéma souvent invoqué de Karl Bühler, qui garde d'ailleurs son utilité ${ }^{36}$. Les expériences de Osgood ont montré que la connotation, qu'il appelle malheureusement meaning tout court, n'est pas stable : il faudrait cependant qu'elle le soit, pour qu'on arrive à une véritable structure. Je crois qu'il faut prendre au sérieux ces résultats expérimentaux. Le behaviorisme linguistique est souvent beaucoup plus raffiné que ne le croient les générativistes ${ }^{37}$.

La sémantique contemporaine a atteint un degré de raffinement impressionnant, mais c'est un raffinement à l'intérieur et dans les limites de l'«esprit de géométrie ». Ce qui serait nécessaire, à mon avis, ce serait un raffinement dans le sens de 1'《esprit de finesse ». Les "principes nets et grossiers (!) de géométrie, dit Pascal, sont palpables, mais éloignés de l'usage commun. Mais dans l'esprit de finesse, les principes sont dans l'usage commun et devant les yeux de tout le monde (!) [...] il n'est question que d'avoir bonne vue (!), mais il faut l'avoir bonne : car les principes sont si déliés et en si grand nombre, qu'il est presque impossible qu'il n'en échappe (!) [...] On les voit à peine, on les sent plutôt qu'on ne les voit; on a des peines infinies à les faire sentir à ceux qui ne les sentent pas d'eux-mêmes : ce sont choses tellement délicates et nombreuses, qu'il faut un sens bien délicat et bien net pour les sentir, et juger droit et juste selon ce sentiment (!), sans pouvoir le plus souvent les démontrer par ordre comme en géométrie, parce qu'on n'en possède pas ainsi les principes (!), et que ce serait une chose infinie de l'entreprendre. Il faut tout d'un coup voir la chose d'un seul regard (!), et non par progrès de raisonnement, au moins jusqu'à un certain

36. K. Bühler, Sprachtheorie, Die Darstellungsfunktion der Sprache, $2^{e}$ éd., Stuttgart, G. Fischer, 1965.

37. C. E. Osgood, G. J. Suci et P. H. Tannenbaum, The Measurement of Meaning, Urbana (III), 1957. 
degré... ${ }^{38} »$. Ce fragment génial esquisse, d'une manière précise, les procédés et l'attitude d'esprit, la forma mentis, requis pour la description de ces éléments les plus élusifs - et les plus importants - d'une langue; il met en relief aussi, d'une manière très claire, une des difficultés de la structuration sémantique du lexique : la notion de «structuration », telle que nous l'avons définie au début, n'est-elle pas liée, étroitement et inévitablement, au type d'esprit caractérisé par Pascal comme «esprit de géométrie »? Le rôle important que jouent, dans la sémantique moderne, les modèles géométriques - les triangles, les cercles, les trapèzes, les champs - n'est-il pas symptomatique à cet égard ${ }^{39}$ ? Deux précisions cependant sont nécessaires; d'abord : ce que Pascal appelle « esprit de finesse 》 (ou encore « esprit de justesse ») est tout autre chose qu'un abandon du rationnel, cet esprit n'est pas moins rationnel que l'autre ${ }^{40}$; ensuite : l'absence de $l^{\prime}$ ' esprit de géométrie »n'entraîne pas automatiquement la présence de l'«esprit de finesse », celui-ci presuppose au contraire un effort sui generis, car «les esprits faux ne sont jamais ni fins ni géomètres ».

J'avoue volontiers que ce que je viens de dire a l'air d'un «credo»: il faudrait, évidemment, plus de concrétion et une base empirique assez large, pour développer ce que je n'ai pu qu'esquisser. D'autre part, je ne peux me soustraire à l'impression qu'il y a, à la base de tous ces efforts prolongés, toujours renouvelés pour en arriver à une structuration dans le lexique, une espèce de credo, quelque chose comme un obstiné e pur si muove. J'ai l'impression aussi, que le Galilée de la sémantique n'est pas encore entré en scène. Lyons déclare sèchement : « No one has yet presented even the outlines of a satisfactory and comprehensive theory of semantics ${ }^{41}$. $\gg$ Quand on parle des difficultés de la structuration sémantique du lexique, on ne peut, en effet, passer sous silence que le nombre des théories sémantiques structurales est presque égal au nombre des linguistes qui ont travaillé sur ce sujet : Trier, Weisgerber, Porzig, Hjelmslev, Pottier, Greimas, Leisi, Coseriu, Prieto, Baldinger, Heger, Rodríguez Adrados, Hilty, Lounsbury, Bendix, Weinreich, Lyons, Hattori, Katz, Ullmann, Osgood, etc., quot homines, tot semanticae! En même temps, le nombre des exemples expliqués est singulièrement réduit.

Il ne s'agit pas de condamner d'une façon sommaire l'approche structurale dans le domaine lexical ; telle n'est point du tout notre intention, qui se veut plus interrogative qu'affirmative. Nul doute que cette approche a enrichi considérablement - et enrichira encore - nos connaissances, car elle n'est pas simplement "fausse», elle contient, au contraire, comme nous l'avons indiqué, d'importants éléments de vérité : la description doit se concentrer en effet sur les multiples relations entre les contenus lexicaux, elle y doit tenir compte, d'une manière non

38. B. Pascal, Pensées, éd. Brunschvicg, Paris, Garnier, 1961, S. 73/74.

39. Cf. H. Henne et H. E. Wiegand, "Geometrische Modelle und das Problem der Bedeutung 》 dans Zeitschrift für Dialektologie und Linguistik, 36, 1969, p. 129-173.

40. Curieusement le mot "justesse " revient dans le titre d'un des livres lexicologiques les plus importants du xviI siècle, la première "synonymique " européenne, celle de $G$. Girard : la Justesse de la langue française ou les différentes significations des mots qui passent pour synonymes (Paris, 1718) ; dans ce livre, qui eut un succès considérable, Girard fait amplement preuve de son «esprit de finesse ; cf. H.-M. Ganger, Die Anfänge der Synonymik : Girard und Ronhaud. Ein Beitrag zur Geschichte der lexikalischen Semantik, Tübingen, 1970 (à paraître).

41. J. Lyons, Introduction to Theoretical Linguistics, Cambridge, UP, 1968, p. 402. 
dogmatique, du principe de l'opposition, et elle doit, en faisant cela, analyser ces contenus en unités plus petites, en sèmes, qui doivent être définis avec la plus grande précision, précision-finesse, possible. En tant que projet global et exclusif cependant, l'approche structurale, dans le champ du lexique, me paraît chimérique et fausse. Prise dans ce sens-là, elle ne peut être sauvée en y « ajoutant » simplement quelques éléments qui lui manqueraient, car elle n'est pas, en tant que projet global, incomplète ou partiale : elle est fausse. Elle est fausse parce qu'elle part d'une conception erronée du contenu lexical. De toute façon, la longue expérience structurale, dans le champ du sémantisme lexical, aura été utile, non seulement, mais très certainement dans le sens qu'a indiqué une fois un des founding fathers de la linguistique moderne : «There is perhaps no better way to learn the essential nature of speech than to realize what it is not and what it does not do ${ }^{42}$.

Hans-Martin Gauger

\section{QUESTIONS}

$M$. Coseriu : J'ai suivi avec beaucoup d'intérêt l'exposé de M. Gauger parce qu'il est intéressant d'apprendre ce qu'on pense dans des milieux de linguistes qui ne se sont jamais occupés de la sémantique structurale, ce qu'on y pense de la linguistique et de la sémantique structurales. Quant aux difficultés qu'il a signalées, ces difficultés peuvent être interprétées dans deux sens différents. Ou bien il s'agit des difficultés que les linguistes appartenant à ce milieu imputent aux structuralistes, ou bien il s'agit des difficultés que ces linguistes ont eux-mêmes à déchiffrer en sémantique structurale. Dans le premier cas, l'on peut rassurer M. Gauger, ces difficultés sont inexistantes parce que le structuralisme sérieux s'occupe du fonctionnement effectif du langage; il n'impose pas de structures au langage, il constate les structures qui sont là effectivement et, dans ce sens, le structuralisme est une constatation, il n'est pas une hypothèse. $\mathrm{Ce}$ que $\mathrm{M}$. Gauger a soutenu constitue une hypothèse et une hypothèse invérifiable, elle est en réalité absurde. Si on interprète ces difficultés dans le deuxième sens, alors oui, il faut bien admettre que ces difficultés existent effectivement. Mais dans ce cas, ces difficultés sont hélas subjectives; on n'a pas de raison de les attribuer à l'objet. Je voudrais ajouter encore trois observations concernant des points particuliers. Cela n'est pas vrai tout simplement que la distinction entre signification et désignation se fonde sur une prétendue hypothèse struc- turale et qu'elle constitue par là une petitio principii. La distinction, on le sait bien, est beaucoup plus ancienne que la reconnaissance des structures et l'on constate tout d'abord qu'il y a une différence entre la signification et la désignation et ensuite l'on constate que la signification est en partie structurée et structurable tandis que la désignation ne l'est pas. Par conséquent la petitio principii n'existe que dans l'interprétation et non pas dans la notion même et dans la distinction même entre signification et désignation. C'était une petitio principii très étrange parce qu'elle implique en même temps une ignoratio hellenchi, ceci pour ceux qui ont des oreilles grecques pour entendre. Deuxième observation, en ce qui concerne Freud, on s'est occupé de Freud et de sa théorie du langage dans un séminaire interdisciplinaire à Tübingen avec des psychologues, des médecins, des philosophes, pendant deux semestres. La conclusion a été la suivante : la théorie du langage de Freud est une théorie péniblement pauvre, péniblement élémentaire, il n'y a absolument rien à utiliser dans cette théorie. Troisième observation concernant la compétence communicative die kommunikative Kompétenz de Habermas, M. Gauger l'a présentée comme s'il s'agissait d'une compétence supérieure, englobant la compétence linguistique. Or chez Habermas il s'agit en réalité d'une étrange compétence à côté de la compétence linguistique. Il l'oppose à la compétence linguistique de Chomsky, qu'il n'a pas

42. E. Sapir, Language, An Introduction to the Study of Speech, New York, 1949, p. 219. 
compris du reste, et il attribue à la compétence communicative une partie de la compétence linguistique qui est parfaitement linguistique dans le sens de Chomsky, et qui est, dans ce cas, la vraie. Je ne suis pas chomskyen on le sait bien. Par conséquent, la compétence communicative de Habermas révèle tout simplement une incompétence linguistique manifeste.

Réponse : Je ne sais pas si je peux reprendre point par point votre contre-exposé, $M$. Coseriu. Je vous remercie de toute façon de votre intervention et si mon exposé a eu quelques mérites, celui d'avoir provoqué votre intervention n'est certainement pas le moindre. La position de M. Coseriu, vous l'avez vu, est extrêmement claire ; il dit : de deux choses l'une, ou vous acceptez la théorie que je défends ou vous cessez, s'il voưs plaît, de faire de la linguistique. Cette alternative me paraît simplement un peu exagérée. Quant aux points particuliers, il faudrait en parler longtemps. Je veux bien croire que vous ayez eu les résultats dont vous avez parlé, mais vous savez qu'il y a d'autres penseurs qui pensent d'une manière tout à fait différente. Il y a d'autres témoins. Je crois qu'il y a à côté de certains séminaires, qu'on fait à Tüibingen et auxquels j'ai participé très souvent moi-même, d'autres autorités. Je suis, quant au milieu dans lequel je me meus, un élève de $M$. Coseriu, bien qu'un peu contestataire comme vous l'avez vu. Je ne suis pas du tout d'accord avec Habermas, c'est seulement l'expression "compétence communicative» que j'ai citée. Le concept de compétence linguistique est un concept qui chancelle. Beaucoup ne croient plus en ce concept et j'ai pensé que cette expression "compétence communicative $"$, pourrait remplacer le concept de compétence linguistique qui est certainement trop étroit. Il n'y a pas une compétence linguistique qu'on peut séparer des compétences beaucoup plus générales. 